\title{
Radio Spectral Index Analysis and Classes of Ejection in LS I $+61^{\circ} 303$.
}

\author{
M. Massi \\ and \\ M. Kaufman Bernadó ${ }^{1}$ \\ Max Planck Institut für Radioastronomie, Auf dem Hügel 69, 53121 Bonn, Germany \\ mmassi@mpifr-bonn.mpg.de
}

\begin{abstract}
LS I $+61^{\circ} 303$ is a $\gamma$-ray binary with periodic radio outbursts coincident with the orbital period of $P=26.5 \mathrm{~d}$. The origin of the radio emission is unclear, it could be due either to a jet, as in microquasars, or to the shock boundary between the Be star and a possible pulsar wind. We here analyze the radio spectral index over $6.7 \mathrm{yr}$ from Green Bank Interferometer data at $2.2 \mathrm{GHz}$ and $8.3 \mathrm{GHz}$. We find two new characteristics in the radio emission. The first characteristic is that the periodic outbursts indeed consist of two consecutive outbursts; the first outburst is optically thick, whereas the second outburst is optically thin. The spectrum of LS I $+61^{\circ} 303$ is well reproduced by the shock-in-jet model commonly used in the context of microquasars and AGNs: the optically thin spectrum is due to shocks caused by relativistic plasma ("transient jet") traveling through a pre-existing much slower steady flow ("steady jet"). This steady flow is responsible for the preceding optically thick spectrum. The second characteristic we find is that the observed spectral evolution, from optically thick to optically thin emission, occurs twice during the orbital period. We observed this occurrence at the orbital phase of the main $26.5 \mathrm{~d}$ outburst and also at an earlier phase, shifted by $\Delta \Phi \sim 0.3$ (i.e almost 8 days before). We show that this result qualitatively and quantitatively agrees with the two-peak accretion/ejection model proposed in the past for LS I $+61^{\circ} 303$. We conclude that the radio emission in LS I $+61^{\circ} 303$ originates from a jet and suggest that the variable $\mathrm{TeV}$ emission comes from the usual Compton losses expected as an important by-product in the shock-in-jet theory.
\end{abstract}

\footnotetext{
${ }^{1}$ Humboldt Research Fellow
} 
Subject headings: Radio continuum: stars - X-rays: binaries - gamma-rays: observations - X-rays: individual: LSI+61303

\section{Introduction}

The TeV-emitting source LS I $+61^{\circ} 303$ is a high-mass X-ray binary system, where a compact object travels through the dense equatorial wind of a Be star. Due to the large uncertainty in the inclination of the system $\left(i=30^{\circ} \pm 20^{\circ}\right)$ the nature of the compact object has not yet been determined and either a neutron star or a black hole are equally plausible candidates (Casares et al. 2005). The most typical peculiarity of LS I $+61^{\circ} 303$ is a large periodic radio outburst (Taylor \& Gregory 1982) with a period of $P_{1}=26.496 \mathrm{~d}$ which is modulated by a period of $P_{2}=1667 \mathrm{~d}$ (Gregory 2002). The $26.5 \mathrm{~d}$ periodicity corresponds

to the orbital period of the binary system (Hutchings \& Crampton 1981). The origin of the second period is unclear. Observed also in the $\mathrm{H} \alpha$ emission line (Zamanov \& Martí 2000), this long period has been related to possible variations in the equatorial wind of the Be star (as a periodic shell ejection, Gregory \& Neish 2002). However, the $\mathrm{H} \alpha$ emission presents drastic variations in less than $24 \mathrm{~h}$ more likely originating from a variable source of ionization (like a relativistic jet) than from improbably drastic changes in the density properties of the Be star disk (Grundstrom et al. 2007).

High resolution radio images of LS I +61³03 have generated controversial interpretations. The bottom image in Fig. 1 shows an elongated structure, which is assumed to be the cometary tail predicted at the shock boundary between the wind from the Be star and the relativistic wind of a pulsar (Dhawan et al. 2006). Alternatively, in the context of the microquasar model, there are two extreme substructures in the same image of Fig. 1 which can be interpreted as two ejections around a re-formed steady jet. The different distances of these substructures from the core as well as the assymmetry seen in the central elongated feature could be taken as the evidence for Doppler boosting effects in the jet (Massi 2007 and Sec. 4.2). Applying the two models, structures in different images appearing at different position angles are taken as a cometary tail always pointed away from the Be star (Dhawan et al. 2006) or as a precessing jet (Massi 2007 and references therein). Taking into account the morphology of these high resolution VLBA images it is not possible to distinguish between the two scenarios. Therefore a more decisive physical parameter is needed to decide between these two possible models.

The physical process producing the radio emission in both cases is the synchrotron mechanism. However, the evolution of the spectrum corresponding to the emission from a bubble of electrons in expansion is quite different from the evolution of a spectrum from a 
relativistic jet with internal shocks as shown in the comparison of the bubble model vs. the shock model by Hannikainen et al. (2006). To distinguish between the microquasar and the pulsar model we therefore decided to study the spectral index accompanied by an analysis of the flare amplitudes at different frequencies. Sections 2 and 3 we present 6.7 years of Green Bank Interferometer (GBI) data and the results of our analysis. In Sect. 4 and Sect. 5 we show that the radio spectral index is a very powerful tool that complements the astrometry and X-ray observations. Finally, in Sect. 6, we summarize our conclusions.

\section{Green Bank Interferometer Radio Data}

We here analyze 6.7 years of the NASA/NRAO Green Bank Interferometer (GBI) LS I $+61^{\circ} 303$ database at $\nu_{1}=2.2 \mathrm{GHz}$ and $\nu_{2}=8.3 \mathrm{GHz}$. The database covers three periods: 49379.975-50174.710 MJD, 50410.044-51664.879 MJD and 51798.333-51823.441 MJD. The samples of each period are continuous with at least two observations per day at each frequency. Together with the flux densities at $\nu_{1}$ and $\nu_{2}$ and their corresponding errors, the GBI database also provides the spectral index, $\alpha=\frac{\log \left(S_{1} / S_{2}\right)}{\log \left(\nu_{1} / \nu_{2}\right)}$. We calculated the error $\Delta \alpha$, as $\Delta \alpha=\frac{0.434}{\log \left(\nu_{1} / \nu_{2}\right)} \sqrt{\left(\frac{\Delta S_{1}}{S_{1}}\right)^{2}+\left(\frac{\Delta S_{2}}{S_{2}}\right)^{2}}$ and the weighted average $\langle\alpha\rangle$ in each phase bin as $\frac{\Sigma \frac{\alpha_{i}}{\Delta \alpha_{i}^{2}}}{\Sigma \frac{1}{\Delta \alpha_{i}^{2}}}$ with $\sigma=\sqrt{\frac{1}{\Sigma \frac{1}{\Delta \alpha_{i}^{2}}}}$. In Figs. 2- a and 2-c we show the whole dataset at $8.3 \mathrm{GHz}$, first folded with the long term period of $P_{2}=1667 \mathrm{~d}$ and then folded with the orbital period of $P_{1}=26.496 \mathrm{~d}$. The phase $\Theta$ refers to the fractional part of $\frac{\left(t-t_{0}\right)}{P_{2}}$, whereas the orbital phase, $\Phi$ refers to the fractional part of $\frac{\left(t-t_{0}\right)}{P_{1}}$, with $t_{0}=J D 2443366.775$ (Gregory 2002).

\section{Results}

Figure 2-b shows the evolution of the spectral index, $\alpha$, during the $1667 \mathrm{~d}$ cycle. A remarkable difference is obvious: the negative values of $\alpha$, i.e. optically thin emission, correspond to a minimum in the flux density, whereas towards the maximum of the cycle $\alpha$ can also be zero or positive, i.e. the spectrum can be flat or inverted.

In Fig. 2-d, the 6.7 yr dataset is folded with $P_{1}$. This figure reveals that the spectrum is flat or inverted in the interval $\Phi \sim 0.22-0.35$ and more clearly in the interval $\Phi \sim 0.46-0.7$. At $\Phi \sim 0.7, \alpha$ declines very rapidly toward a value of $\alpha \simeq-0.4$. In the following subsections, we will show that this evident transition of the spectral index is an important tool for distinguishing between the microquasar and the pulsar model. 


\subsection{Pulsar vs. Shock-in-Jet Model}

Two types of radio emission are observed from X-ray binaries with jets. They have completely different characteristics and are associated with different kinds of ejections. One corresponds to a flat or inverted spectrum and the other to an optically thin spectrum (Fender et al. 2004). The flat or inverted spectrum covers the whole radio band and has been established also at millimeter and infrared wavelengths (Fender et al. 2000; Fender 2001; Russel et al. 2006). When this kind of radio emission is spatially resolved it appears as a continuous jet, the so-called "steady jet". In contrast, the optically thin spectra are associated with isolated components, so-called "knots" or "plasmoids", which are moving at relativistic speed away from the binary. This kind of ejecta are also known as a "transient jet" (Fender et al. 2004, 2006).

The most important point is that the two kinds of radio emission and their corresponding types of ejection are related to each other; if the optically thin spectrum (the transient jet) appears, then it always happens after the flat/inverted one (the steady jet). Fender et al. (2004) associate this change in the radio spectrum to the parallel change that is observed in the X-ray states of these sources if passing from the low/hard X-ray state to the steep power-law state. In such a passage there is an increase in the bulk Lorentz factor of the jet. This increase gives rise to shocks where the new highly-relativistic plasma catches up with the pre-existing slower-moving material of the steady jet. Fender et al. (2004) extended the AGN-shock model to all the X-ray binary-jet-systems (i.e. microquasars). That model was originally derived by Marscher \& Gear (1985), then generalized by Valtaoja et al. (1992), and introduced in the context of X-ray binaries for GRS 1915+105 by Kaiser et al. (2000). This internal shock model predicts a clear trend in flux variations depending on frequency, which will be described below.

The transition between the two kinds of radio emission (optically thick to optically thin) therefore is an important characteristic of microquasar systems. This feature is clearly shown in our data presentation. Figure 3 shows the two light curves and spectral index of data at $\Theta=0.0-0.1$ (1455 measurements at each frequency) folded with the orbital period $P_{1}$. That figure shows a main peak of $S_{8.3 \mathrm{GHz}}=270 \pm 15 \mathrm{mJy}$ at $\Phi=0.69$. We call this outburst $P e a k_{1}$, which is an optically thick outburst, as one can determine from the clear positive spectral index shown in the figure (Top). At $2.2 \mathrm{GHz}$ the figure shows that $P_{e a k_{1}}$ is followed by a large outburst at $\Phi=0.82$; we call this $P e a k_{2},\left(S_{2.2 \mathrm{GHz}}=299 \pm 6 \mathrm{mJy}\right)$. At 8.3 GHz Peak $\mathrm{F}_{2}$ corresponds to a minor outburst resulting in a clear optically thin spectrum. In Fig. 3-Top the spectral index declines between the two peaks with an inversion of the spectrum from optically thick to optically thin at $\Phi \simeq 0.75$. After Peak $k_{2}$ the spectral index remains rather constant at a value of $\alpha \simeq-0.4$. In Fig. 4.Top we analyze individual light 
curves (a 26 d data set, 126 measurements at each frequency) selected at a different phase of $P_{2} \Theta=0.76$. The curve at $2.2 \mathrm{GHz}$ clearly shows the two consecutive peaks. At $\Phi \simeq$ 0.50 we have Peak 1 with $S_{8.3 \mathrm{GHz}}=230 \pm 13 \mathrm{mJy}$ and $S_{2.2 \mathrm{GHz}}=214 \pm 5 \mathrm{mJy}$. At $\Phi=0.61$ both frequencies have a minimum, and at $\Phi \simeq 0.68$ Peak $_{2}$ follows with $S_{8.3 \mathrm{GHz}}=193 \mathrm{mJy}$ and $S_{2.2 \mathrm{GHz}}=269 \mathrm{mJy}$. The averaged spectral index for the interval $\Theta=0.7-0.8$, shown in Fig. 5 panel 7, again clearly shows the decline of $\alpha$ between the two outbursts $(\Phi=0.5-0.7)$. In Fig. 4-Bottom we analyze other individual light curves at $\Theta=0.85$ showing a yet better case, with more displaced $P e a k_{1}$ and $P e a k_{2}$ with an intermediate, additional optically thick peak. In the same plot (Fig. 4.Bottom) the $\mathrm{H} \alpha$ emission-line measurements by Grundstrom et al. (2007) are given. The $\mathrm{H} \alpha$ value, still high at $\Phi=0.71$, shows a dramatic decline at $\Phi=0.749$ (1 day later) corresponding with the onset of the optically thin outburst (see discussion in Grundstrom et al. 2007).

In other words, the light curves indicate that there is not only a single outburst appearing after some delay at different frequencies, but that there are two distinct outbursts at both frequencies. The physics behind the two outbursts seems to be quite different. In the first outburst the spectrum is flat or inverted, whereas the second outburst is clearly dominated by the peak at $2.2 \mathrm{GHz}$ (optically thin outburst). The optically thin outburst corresponds to different conditions, as its much higher amplitude, the spectral index, and the $\mathrm{H} \alpha$ emissionline measurements indicate.

Let us first examine the pulsar model. The variation of the spectrum from inverted to optically thin would correspond to an expanding synchrotron-emitting region in any model. In the pulsar scenario it would be the two colliding winds of a pulsar and Be star. We can assume that the initial region, sufficiently compact to be optically thick, afterwards expands and becomes optically thin. However, following van der Laan (1966) this model predicts that the self-absorption turnover peak frequency will move to lower frequencies, and the peak flux density will diminish (Valtaoja et al. 1992; Hannikainen 2006). In other words, this model predicts only one outburst at each frequency, moving to lower frequencies with lower flux densities. Considering that in fact there are two outbursts, the adiabatic expansion could explain the small delay observable between $P e a k_{1}$ at $8.3 \mathrm{GHz}$ and $2.2 \mathrm{GHz}$, i.e. the optically thick outburst, but cannot explain why the flux would rise again producing Peak ${ }_{2}$.

On the other hand, the two interacting winds of the pulsar model suggest a prolonged injection of energetic particles, rather than a single event. Connors et al. (2002) have shown that a prolonged injection of energetic particles can maintain an outburst optically thin, as is observed for the pulsar PSR B1259-63, which is in an orbit around a Be star. In PSR B1259-63 the spectrum is optically thin throughout the outburst as is demonstrated in Fig. 3 of Connors et al. (2002). In Fig. 2 of Paredes et al. (1991) is shown that a prolonged 
injection of energetic particles, can reproduce only the optically thin outburst $\left(P_{2} a k_{2}\right)$ of LS I $+61^{\circ} 303$. Therefore, the model with prolonged injection of energetic particles cannot explain why an optically thick Peak ${ }_{1}$ should be present in LS I $+61^{\circ} 303$ before Peak ${ }_{2}$.

The complex peak sequence that we found in LS I $+61^{\circ} 303$ finds a natural explanation in the disc-jet coupling model described by Fender et al. (2004): first there is a continuous outflow with flat or inverted spectrum, then an event (probably Peak 1 ) triggers a shock in this slow optically thick outflow (Fender et al. 2004). At this point the young growing shock creates the optically thin outburst ( Peak 2 ) (Valtaoja et al. 1992; Hannikainen et al. 2006). Like for the observations of the microquasar GRO J1655-40 explained with the shock-in-jet model (Hannikainen et al. 2006), in LS I $+61^{\circ} 303$ one observes the increase of the flare amplitude with decreasing frequency, resulting in an optically thin flare. The agreement between LS I $+61^{\circ} 303$, with spectral index $\alpha \simeq-0.4$, and GRO J1655-40 is impressive. In Hannikainen et al. (2006, Fig. 4), the spectral index, $\alpha$, during the outburst and during the decay remains in the range $\alpha=-0.6$ to -0.4 .

During the stage of "growth" of the shock, the dominant cooling mechanism is inverse Compton losses producing gamma-rays (Marscher \& Gear 1985). Inverse-Compton losses fall off with the radius as the shock expands and are superseded by synchrotron losses. It is interesting to see that this second stage of the shock-in-jet model, where synchrotron losses dominate, is different in different sources. In this phase, after the peak, either the flux density decay begins like in the blazar 3C 279 (Lindford et al. 2006) and LS I +61³03, or the flux density remains roughly constant in a "flat" stage, like in 3C 273 and the microquasar GRS

1915+105 (Türler et al. 2000; 2004). The analogous behaviour of LS I $+61^{\circ} 303$ and the blazar 3C 279 during the second stage is worth of note considering the low inclination angle for the orbit of LS I $+61^{\circ} 303$. Moreover, in another blazar, BL Lac, Marscher et al. (2008) recently found evidence for a double consecutive optical flare. The authors discuss that the first flare corresponds to a disturbance due to explosive activity near the black hole, then the disturbance forms a shock wave responsible for the second flare. A similar interpretation can be applied to the double radio flare of LS I $+61^{\circ} 303$, suggesting that LS I $+61^{\circ} 303$ is a microquasar with a jet close to the line of sight, i.e. a microblazar, where explosive activity and the shock wave are seen almost face on.

\subsection{The Two-Peak Accretion/Ejection Model}

Figure 3-Top shows that the evolution from an optically thick to an optically thin spectrum occurs twice, giving the curve $\alpha$ vs $\Phi$ a double-peaked shape. In Fig. 5 we show the whole 6.7 yr data set, divided in $\Theta$ intervals. The double-peaked curve is confirmed in 
the intervals $\Theta=0.7-0.3$, which corresponds to the maximum of the $1667 \mathrm{~d}$ cycle. In this $\Theta$ interval, the spectrum is flat or inverted $(\alpha \geq 0)$ in the two orbital phase ranges $0.22 \pm 0.08$ to $0.33 \pm 0.12$ and $0.50 \pm 0.09$ to $0.70 \pm 0.13$. Around the minimum of the $1667 \mathrm{~d}$ cycle, at $\Theta=0.4-0.5$, these two intervals, in which the spectrum is inverted, seem to be reduced to only a few points with $\alpha \geq 0$.

Figure 6 shows the data of Fig. 3 (i.e. $\Theta=0.0-0.1$ ) plotted as spectral index vs. flux density. The change from an inverted spectrum to optically thin emission around orbital phase $\Phi \sim 0.75$ occurs at high flux densities. This change has been explained in Sect. 3.1 due to shock propagation throughout the pre-existing slow jet formed during the phase with an inverted spectrum. In contrast, the previous change around orbital phase $\Phi \sim 0.48$ occurs at low flux densities. The similar evolution of the spectrum, flat or inverted spectrum (steady jet) to optically thin jet (transient jet) indicates that the same physical process does indeed occur twice in LS I $+61^{\circ} 303$, but the first time something strongly attenuates the emission.

This hypothesis finds support in the predictions of the two-peak accretion model. Taylor et al. (1992) and Martí \& Paredes (1995) have modeled the properties of LS I +61³03 in terms of an accretion rate $\dot{M} \propto \frac{\rho_{\text {wind }}}{v_{\text {rel }}^{3}}$ (where $\rho_{\text {wind }}$ is the density of the Be star wind and $v_{\text {rel }}$ is the relative speed between the accretor and the wind), which creates two peaks because of the high eccentricity $(e=0.7)$. The highest peak corresponds to the periastron passage, because of the highest density, while the second peak occurs when the drop in the relative velocity $v_{\text {rel }}$ compensates (because of the inverse cube dependency) the decrease in density. Martí \& Paredes (1995) have shown that both peaks are above the Eddington limit and therefore one expects that matter is ejected twice within the $26.5 \mathrm{~d}$ interval (an updated version of the accretion rate curve, for periastron passage at $\Phi=0.23$, is given in Fig. 2 of Bosch-Ramon et al. (2006)).

The two-peak accretion model has found confirmation through the observations in another X-ray binary system, Circinus X-1, which also has a rather high eccentricity, e $=0.8$ (Murdin et al. 1980). The observations by Tudose et al. (2006) show indeed a radio peak at periastron and a second peak around apoastron. The important difference between Circinus $\mathrm{X}-1$ and LS I $+61^{\circ} 303$ is the nature of their respective companion stars. The companion star of Circinus X-1 is clearly cooler than a B5 star (Jonker et al. 2007) whereas in LS I +61³03 it is a B0 V star (Casares et al. 2005). In LS I +61 303 the ejected relativistic electrons near periastron are embedded in such a strong UV-radiation field that they nearly completely loose their energy through inverse-Compton scatterings. This hypothesis found both theoretical and observational support: Bosch-Ramon \& Paredes (2004) proposed a numerical model based on inverse Compton scattering, where ejected relativistic electrons are exposed to stellar photons (external Compton) as well as to synchrotron photons (synchrotron self 
Compton) and found indeed a gamma-ray peak at periastron. The hypothesis of a highenergy outburst at periastron was confirmed (Massi et al. 2005) by observations from the EGRET instrument (i.e., $E>100 \mathrm{MeV}$ ) on board of the Compton Gamma-Ray Observatory satellite (Tavani et al. 1998). Massi et al. (2005) pointed out that the EGRET gamma-ray emission during one full orbit (at JD 2450334, $\Theta=0.18$ ) shows a clear peak at periastron passage and in a previous epoch (at JD 2449045, $\Theta=0.41$ ) shows an increase again near the periastron passage and a second large peak at $\Phi \simeq 0.5$. The inverse-Compton losses that produce the high-energy peak at periastron seem to be severe since in only very few radio light curves can one distinguish a peak at periastron. This is the case in the radio observations that have been simultaneously performed with the MAGIC Cherenkov high-energy telescope. In Fig. S1 of Albert et al. (2006), one can see together with the radio peak at phase $\Phi=0.7$ a second radio peak close to the periastron passage.

The orbital phase of the two EGRET peaks, $\Phi=0.2$ and $\Phi \simeq 0.5$ agree with the two optically thick intervals $0.22 \pm 0.08$ to $0.33 \pm 0.12$ and from $0.50 \pm 0.09$ to $0.70 \pm 0.13$ determined here. The separation between the centers of the two optically thick intervals $(\Phi \simeq 0.3$ and $\Phi=0.6$ ) agrees also with the separation, i.e. $\Delta \Phi=0.3$, between the two accretion peaks of the model by Marti \& Paredes (1995), who adopted parameters for the envelope of the Be star derived from infrared data. Romero et al. (2007) applied a smoothed particle hydrodynamics code to develop three-dimensional, dynamical simulations for LS I $+61^{\circ} 303$ and found that the accretion rate has two peaks per orbit, i.e., a narrow peak at periastron $(\Phi=0.23)$, and a broad peak that lags the periastron passage by about 0.3 in phase. Finally, recent observations with VERITAS ( $E>500 \mathrm{Gev}$, Acciari et al. 2009) give marginal evidence for emission at $\Phi=0.2-0.3$ along with the known emission at $\Phi=0.5-0.9$.

Gamma-ray observations, the two-peak model (fitted to infrared observations) and finally three-dimensional simulations give a separation between the two peaks in agreement with the separation between the intervals we discussed, where the radio spectral index is optically thick. The intervals $(0.22 \pm 0.08$ to $0.33 \pm 0.12$ and from $0.50 \pm 0.09$ to $0.70 \pm 0.13)$ determined here from a database of $6.7 \mathrm{yr}$, therefore lead to important observational support, at radio wavelengths, for the two-peak accretion/ejection model by Taylor et al. (1992) and Martí \& Paredes (1995).

\section{Spectral Index vs. Jet-States}

A telling definition of the two distinct radio emission states, steady jet and transient jet, is radio emission "attached to" and "detached from" the nucleus (Dhawan et al. 2000). In fact, the steady jet (flat or inverted radio emission spectrum) appears as a compact jet 
centered on the engine, whereas the transient jet (emission with an optically thin spectrum) is associated with components moving with relativistic speed away from the binary core (Fender et al. 2004).

In Fig. 5 - $\Theta=0.8-0.9$, we indicate with two bars, where VSOP observations were performed by Taylor et al. (2000, 2001). The VSOP observations occurred within the second optically thick interval. The optically thin outburst, Peak $k_{2}$, connected with the transient jet occurred $24 \mathrm{~h}$ after the end of the VSOP observations.

The implication of this fact is that the set of VSOP images (like that shown in our Fig. 1) used in the past to estimate the "expansion" velocity, show only a core centered, slow, steady jet. This explains the low velocity values derived.

The spectral index analysis therefore is a powerful tool to distinguish between steady and transient jets. We can better prove this by comparing the spectral index analysis with the different displacement of radio structures imaged along the orbit of LS I $+61^{\circ} 303$. To compute such a displacement we use the astrometric results by Dhawan et al. (2006) for their radio features A,B,C,D,E,F,G,H,I and J. We also use the orbit of the system from Casares et al. (2005) and assume that optical and radio frame overlap each other in B.

\subsection{Spectral Index Analysis vs. Astrometry}

The astrometry of the radio peaks of VLBA observations at $3.6 \mathrm{~cm}$ is shown in Fig. 4 of Dhawan et al. (2006). In the figure the orbit is also given as an ellipse of axis $0.5 \mathrm{AU}$ in an arbitrary position. The exact location of the ellipse relative to the radio measurements is, as mentioned by the authors, in fact uncertain.

We adopt for the optical frame the orbit presented in Fig. 2 from Casares et al. (2005), and plot in Fig. 7 7-Top the distance of B (i.e. the orbital phase of B, shown as empty squares) in respect to all other orbital phases corresponding to the VLBA observations. In the same figure we plot the relative displacement (as filled circles) of all the radio peaks reported in Fig. 4 of Dhawan et al. (2006) in respect to B, showing the offsets with an error bar of 0.1 mas, as given by these authors. As a result, the distance between each circle and its relative square gives an estimate of the displacement of the radio structure from the orbit. The result of this comparison is that the radio peaks $\mathrm{C}$ and $\mathrm{J}$ are found to be on the orbit whereas the radio peak $\mathrm{A}$ is clearly offset (by $5 \sigma$ ) in the same way as E,F,G and $\mathrm{H}$. The offsets of the two radio peaks $\mathrm{D}$ and $\mathrm{I}$, displaced $<3 \sigma$ away from the expected orbital position, are not significant for the analysis. In Fig 7 7 Bottom we show $\alpha$ vs $\Phi$ for the subset of the GBI database, $\Delta \Theta=0.32-0.35$, that corresponds to the interval of the Dhawan et al. (2006) 
observations, but at other epochs. Considering that the data refer to a different epoch the agreement between the two plots is rather good. The orbital phase interval, where we find emission centered on the orbit (i.e. B,C and J), is where $\alpha \geq 0$ (i.e. a steady jet). Otherwise the emission clearly is optically thin for the rest of the orbit and this corresponds to the orbital phases of E,F,G and H.

\subsection{Relativistic Doppler Effects}

The radio peak $\mathrm{A}$ is offset $(5 \sigma)$ and the spectral index of Fig.7, even if not at the same epoch of the Dhawan et al (2006) observations, shows indeed minor transients in the optically thick interval. This scenario finds confirmation in the Dhawan et al. (2006) image C (see Fig(1), where components, detached from the nucleus, are observed at the extremes of an already reformed steady jet.

A central instability driving shock waves into a bipolar outflow should create two symmetrical components of shocked material; the lack of a receding component for the radio structures A, E,F,G and H indicates a large Doppler de-boosting factor. Doppler effects are noticeable in $\mathrm{C}$ (here Fig. 1): not only the reformed steady jet is clearly asymmetric, but consistently also the distance of the "plasmoids" from the core is different, with $d_{a}$ for the approaching component larger than $d_{r}$ for the receding component. Inserting the specific values that we get from Fig. 1 into the following relation (Paredes et al. 2002):

$$
\beta \cos \theta=\frac{\left(d_{\mathrm{a}}-d_{\mathrm{r}}\right)}{\left(d_{\mathrm{a}}+d_{\mathrm{r}}\right)},
$$

we derive $\beta \cos \theta \simeq 0.3$. This implies a minimum value $\beta \simeq 0.3$ for small values of $\theta$, the angle between the jet and the line of sight.

With respect to the morphology of the structures we note, that even though some of them may still look like centered one-sided steady jets, this is not in contradiction with the fact that they are far away from the center of the system, instead. This kind of morphology was also found in the microquasar GRS $1915+105$ and can be seen in the last image of Fig. 2 from Fender et al. (1999), which clearly shows two-sided ejections (C2, C3, and NW), where the offset structures look like one-sided jets very similar to the radio structure $\mathrm{F}$ at $\Phi=0.600$ in Fig. 3 from Dhawan et al. (2006). That means the morphology can be rather misleading. 


\section{Spectral Index vs. X-ray States}

Steady jets and transient jets, present in microquasars, are produced when the X-ray binary is in one of the two different X-rays states: low/hard and steep power-law state (Fender et al. 2004). The low/hard X-ray state is the state characterized by a power-law with photon index $1.5<\Gamma<2.1$ (Remillard \& McClintock 2006) and a clear steep cutoff near $100 \mathrm{keV}$ (Grove et al. 1998). The steep power law X-ray state (formerly called very high state) was renamed after monitoring programs of $R X T E$ showed that, whereas an unbroken steep power-law is a fundamental property of the state, a very high luminosity is not. The photon index in the X-ray and gamma-ray bands is the same: $\Gamma \sim 2.5-3.0$ (McClintock \& Remillard 2006).

\subsection{Optically Thin Radio Spectrum and Steep Power-Law X-ray State}

Whereas there are MAGIC observations where LS I $+61^{\circ} 303$ remains undetected, it is significant that when it is detected, the energy spectrum is always well fitted by a power-law with photon index equal to $\Gamma \simeq 2.6$. Albert et al. (2008a,b) discuss that although there is clear evidence for a significant change in the flux level for two observations at two different epochs and at $\Phi=0.5-0.6$ and $\Phi=0.6-0.7$, the photon index during the first observation was $\Gamma=2.7 \pm 0.4$ and for the second observation was $\Gamma=2.6 \pm 0.2$. This is consistent with the steep power law state, where the photon index is a fundamental property of the state and not the luminosity (McClintock \& Remillard 2006). Chernyakova et al. (2006) analyzed INTEGRAL data from the time interval 2003-2005. This interval corresponds to $\Theta$ from $\sim 0.6$ to $\sim 1.0$, i.e. during the maximum of the $1667 \mathrm{~d}$ cycle, where the two optically thick intervals cover the phases from $\Phi=0.2 \pm 0.1$ to $0.6 \pm 0.1$. A steep power-law X-ray state is expected where $\alpha$ declines under zero, i.e. after $\Phi=0.6 \pm 0.1$. Indeed, in the orbital phase interval $\Phi=0.6-0.8$, Chernyakova et al. (2006) determine $\Gamma=3.6_{-1.1}^{+1.6}$. If $\Phi=0.4-0.6$ they determine $\Gamma=1.7 \pm 0.4$ and if $\Phi=0.8-0.4 \Gamma=1.4 \pm 0.3$. EGRET observations at $\Theta=0.18$, shows the peak predicted by the two-peak accretion model at periastron passage. However, EGRET data at $\Theta=0.4$, like MAGIC observations, show a second broad peak at $\Phi \simeq 0.5$, as would be expected between X-ray and very high energy, in the case of an unbroken power law. MAGIC observations simultaneous with INTEGRAL observations are not available and therefore fluxes cannot be extrapolated and compared. However, it is a fact that the measured photon indices in the X-ray and gamma-ray bands overlap the expected range for the steep power-law state and that this occurs in the orbital range where, following our spectral index a steep power-law state is expected.

Simultaneous observations of MAGIC and INTEGRAL exist for Cygnus X-1 (Malzac 
et al. 2008 and reference there). The photon index of MAGIC observations fitted by a power-law is $\Gamma=3.2 \pm 0.6$. INTEGRAL observations have only be fitted with a power-law model plus cut-off (i.e. as a low-hard) and therefore cannot be compared. However, the very important result is that $\mathrm{TeV}$ emission can be extrapolated down to the $\mathrm{MeV}$ range assuming $\Gamma=2.5$ (Fig. 6 in Malzac et al. 2008). The transition in Cygnus X-1 from its low X-ray state and "high X-ray state" is a transition from low-hard state with a breaking type spectrum with $\Gamma \sim 1.8$, and a steep power-law state with $\Gamma=2.6$ (Figs. 2 and 3 in McConnel et al. 2001). The steep power-law state is "weird" with its low luminosity and the absence of QPO (Mc Clintock and Remillard 2006 and references there).

A steep power-law state could be the origin of the TeV-emission for both LS I $+61^{\circ} 303$ and Cygnus X-1. The physical process could be synchrotron self Compton scattering by the shock-generated population of relativistic electrons responsible for the radio emission. In Fig. 3 the spectral index at the peak of the optically thin outburst, Peak $k_{2}$ is $\alpha \simeq-0.5$. Bosch-Ramon \& Paredes (2004) calculated that an electron power-law index $p=1.7$, and therefore $\alpha=-(p-1) / 2=-0.35$, agrees with a photon index $\Gamma=2.2$. The steeper power law $p=2$, resulting from our $\alpha \simeq-0.5$, implies a steeper photon index, i.e. $\Gamma>2.2$, in agreement with the values of the most sensitive observations performed by MAGIC and VERITAS $(2.6 \pm 0.2$ and $2.4 \pm 0.2)$.

\subsection{Inverted Radio Spectrum and Quiescent X-ray State}

The X-ray luminosity $L_{\mathrm{X}}$ in the low/hard X-ray state corresponds to a radiatively inefficient, "jet-dominated" accretion mode (Fender et al. 2003). In this mode only a negligible fraction of the binding energy of the accreting gas is directly converted into radiation and most of the accretion power emerges in kinetic form, as shown for Cygnus X-1 by Gallo et al. (2005), and for AGNs through the relationship between the Bondi power and the kinetic luminosity (Merloni \& Heinz 2007 and references therein). For a compact object of a few solar masses the low/hard state corresponds to $L_{\mathrm{X}} \sim 10^{36} \mathrm{erg} / \mathrm{sec}$ but may drop to $L_{\mathrm{X}}=10^{30.5}-10^{33.5} \mathrm{erg} / \mathrm{s}$ (McClintock \& Remillard 2006) at its lowest phase, called quiescent state. The quiescent state has several characteristics of the low/hard state including the presence of a continuous radio jet. An example of such a situation is the radio jet present in the black hole A0620-00 during its quiescent state (Gallo et al. 2006). There is a relationship between the X-ray and radio luminosities of low/hard state black hole X-ray binaries, that is valid up to X-ray luminosities of $10^{-8.5} L_{\text {Edd }}$ (Gallo et al. 2003, 2006). This important non-linear scaling between X-ray and radio emission has been demonstrated to hold - with the addition of a mass term - across the entire black hole mass spectrum, from microquasars 
to AGN (Merloni et al. 2003; Falcke et al. 2004). The X-ray luminosity of LS I $+61^{\circ} 303$, $L_{\mathrm{X}} \simeq(1-6) \times 10^{34} \mathrm{erg} / \mathrm{sec}$ (Paredes et al. 1997), is between the quiescent and the low $/$ hard states. We checked if such a low luminosity, $L_{\mathrm{X}} \simeq 3 \times 10^{34} \mathrm{erg} / \mathrm{sec}$, correlates with the radio emission $L_{\mathrm{R}}=10^{31} \mathrm{erg} / \mathrm{s}$ (Combi et al. 2004), measured outside the large radio outburst window. The universal relationship for an object of about three solar masses predicts (Merloni et al. 2003):

$$
\log L_{\mathrm{R}}=\eta \log L_{\mathrm{X}}+7.7
$$

with $\eta$ in the range from 0.5 to 0.7 . Substituting the observed luminosities of LS $\mathrm{I}+61^{\circ} 303$ in radio and X-ray, $L_{\mathrm{R}}$ and $L_{\mathrm{X}}$, we obtain a value of $\eta$ consistent with the given range, i.e. $\eta=0.67$. Assuming an average value of $\eta=0.60$, given by Merloni et al. (2203), it would result a radio luminosity of almost three orders of magnitude lower than the observed $L_{\mathrm{R}}=10^{31} \mathrm{erg} / \mathrm{s}$. In other words, LS I $+61^{\circ} 303$ is more radio loud than "average" microquasars (i.e. those corresponding to the center value of $\eta=0.60$ ). In the same way $3 \mathrm{C}$ 273 with $\eta \simeq 0.65$ is more radio loud than AGNs with $\eta=0.60$ (see Fig. 5 by Merloni et al. 2003). LS I $+61^{\circ} 303$ also is extreme concerning other characteristics. Several X-ray binaries show quasi periodic oscillations with timescales of a fraction of minutes during the low/hard X-ray state (Brocksopp et al. 2001). LS I +61³03 shares the remarkable property of extremely slow quasi-periodic oscillations with the black holes V404 Cyg and GRS $1915+105$. During the decay of the 1989 outburst a variety of $22-120 \mathrm{~min}$ oscillations were observed in V404 Cyg (radio: Han \& Hjellming 1992). Quasi periodic oscillations of 30-84 min were observed in LS I +61 303 (radio: Peracaula et al. 1997 and X-ray: Harrison et al. 2000). Radio observations of GRS $1915+108$ by Pooley and Fender (1997) revelead quasiperiodic oscillations with periods in the range $20-40 \mathrm{~min}$ and observations by Rodriguez and Mirabel (1997) oscillations of $30 \mathrm{~min}$.

Besides the low X-ray luminosity the quiescent state has an important characteristic that is perfectly matched by LS I $+61^{\circ} 303$. This important characteristic of the quiescent state is its broadband spectrum with two components: one in the X-ray band (represented by the power law with a photon index of $1.5<\Gamma<2.1$ and a cut-off near 100 $\mathrm{keV}$ discussed above) and the other is in the optical band (see the results from the Hubble Space Telescope and Chandra in McClintock et al. 2003). The observed optical/UV band emission comes predominantly from synchrotron radiation of relativistic electrons present either in the ADAFl (Naravan et al. 1997; McClintock et al. 2003) or in the jet (Russell et al. 2007). In fact, the steady jet is known to radiate at radio/infrared/optical wavelengths (Russell et al. 2006; Russell et al. 2007). In this context the optical observations of LS I $+61^{\circ} 303$ strongly support the hypothesis of a quiescent state. Mendelson \&

\footnotetext{
${ }^{1}$ ADAF: Advection-Dominated Accretion Flow disk model
} 
Mazeh $(1989,1994)$ discovered a clear period $(P=26.4 \mathrm{~d})$ in optical observations. The authors noticed that the optical modulation has the same period as the radio outbursts and rises also in the same orbital phase reaching an amplitude of 0.05 mag. The authors suggested that the minimum is associated with the Be star, whereas the excess is created by a periodic mechanism related with the accretion disk or with optical synchrotron radiation. The photometry in the $\mathrm{V}$ band by Paredes et al. (1994) confirmed the periodicity and the shape of the optical light curve. They found a broad brightness maximum near the major radio outburst, i.e., $\Phi=0.5-0.9$. A new confirmation of the optical modulation comes from the series of observations in the $\mathrm{V}$ and $\mathrm{U}$ bands spanning $6400 \mathrm{~d}$ by Zaitseva \& Borisov (2003). They found the optical period exactly equal to the radio one with a maximum at $\Phi=0.85$ and they noticed that the light curve has a gentler rise and a steeper decline. The correlation between the optical and radio emission of LS I $+61^{\circ} 303$ therefore exists in both period and orbital phase and indicates the same agent: the jet.

\section{Conclusions}

The radio emission from LS I $+61^{\circ} 303$ with periodic $\left(P_{1}=26.5 \mathrm{~d}\right)$ outbursts with an amplitude modulated over $P_{2}=1667 \mathrm{~d}$ is detectable during the whole orbit. If LS I $+61^{\circ} 303$ is a microquasar, the radio emission should show the characteristics of such objects. The current observational picture of jets distinguishes two completly different types of radio emission, one corresponding to a flat or inverted spectrum and the other to an optically thin spectrum (Fender et al. 2004). These two kinds of radio emission and their corresponding types of ejection are related to each other. The optically thin spectrum is created by shocks caused by highly-relativistic plasma (transient jet) traveling through a slower steady-flow (steady jet) established during a previous phase of emission with an inverted spectrum (Fender et al. 2004). If spatially resolved, the flat/inverted spectrum radio emission (steady jet) appears as a continuous jet centered on the system, whereas the optically thin spectrum associated to outbursts (transient jets), is resolved into components ("knots" or "plasmoids") moving with relativistic speed away from the binary core. These two classes of ejection correspond with two different X-ray states. The transient jet is associated with the steep power law X-ray state, i.e. an unbroken steep power-law, where the photon index in the X-ray and gamma-ray bands is the same and equal to $\Gamma \sim 2.5-3.0$. A steady jet corresponds always to the low/hard X-ray state characterized by a power-law with photon index $1.5<\Gamma<2.1$ (Remillard \& McClintock 2006) and a clear steep cut-off near $100 \mathrm{keV}$ (Grove et al. 1998).

We analyzed the radio spectral index using 6.7 years of GBI radio data of LS I $+61^{\circ} 303$. We first compared the radio results with the predictions of two existing alternative models, 
then with astrometric results and finally with available X-ray and gamma-ray observations. Our main conclusions are:

1. The periodic $\left(P_{1}=26.5 \mathrm{~d}\right)$ large radio outburst of $\mathrm{LS} \mathrm{I}+61^{\circ} 303$ indeed consists of two successive outbursts, one optically thick and the other optically thin (Figs. 3 and 4). This peculiar trend does not coincide with an expanding synchrotron-emitting region producing a simple spectrum moving to lower flux densities and lower frequencies. Assuming a prolonged injection of energetic particles one can reproduce the optically thin outburst, but only this one. The fact that a prolonged injection of energetic particles maintains an outburst optically thin has been proved for the pulsar PSR B1259-63, but with the difference to LS I +61³03, that the outburst in PSR B1259-63 remains optically thin throughout. That cannot be compared with the complex sequence (optically thick, optically thin spectrum) of LS I $+61^{\circ} 303$. This complex sequence finds a natural explanation in the shock-in-jet model: first there is a continuous outflow with flat or inverted spectrum, then an event associated with the first outburst and related to a transition of the accretion disc, triggers a shock in the slow, pre-existing optically thick outflow. At this point the young growing shock creates an optically thin outburst, as it is in fact observed in the microquasar GRO J1655-40 and here in LS I +61 303 .

2. During the maximum of the long 1667 d cycle $(\Theta \simeq 1.0)$, we observe that along the $26.5 \mathrm{~d}$ orbit the evolution from an optically thick to an optically thin spectrum occurs twice, giving the $\alpha$ vs $\Phi$ curve a double-peaked shape (Figs. 3 and 5). In particular, the spectrum is optically thick in the interval of orbital phase from $0.22 \pm 0.08$ to $0.33 \pm 0.12$ and again from $0.50 \pm 0.09$ to $0.70 \pm 0.13$. Therefore, during the orbit a transient jet occurs twice, each time preceded by a steady jet. This result agrees, qualitatively and quantitatively with the predictions of the two peak accretion/ejection model, with the results of three-dimensional dynamical simulations and finally with gamma-ray data. All these results indicate a scenario with a first ejection around the periastron passage $(\Phi=0.23)$ with low radio emission, but high energy emission because of inverse Compton losses caused by the proximity of the B0 star, and a second ejection, delayed $\Delta \Phi=0.3$ (i.e. almost $8 \mathrm{~d}$ ) and therefore farther away from the Be star, with negligible losses and well observable at radio wavelengths.

3. We show that the VSOP observations of Taylor et al. (2000) occurred within the second optically thick interval. That implies that the set of VSOP images used to estimate the expansion velocity show only a core-centered slow steady jet and not a transient jet.

4. The observations of Dhawan et al. (2006) with some orbit-centered features along with orbit-displaced features, have been performed at $\Theta=0.32-0.35$. At that $\Theta$ phase 
there exists only the first optically thick interval, i.e. that centered around periastron. It is in this interval that we find the orbit-centered radio structures (i.e. core-centered steady jet). In contrast, the orbital range with only optically thin emission is coincident with the orbital range in which there are radio stuctures displaced from the orbit (i.e shocked "plasmoids").

5. When LS I $+61^{\circ} 303$ was detected with MAGIC (mainly for $\Phi=0.5-0.8$ ), the energy spectrum was always well fitted by a power law with a photon index $\Gamma \simeq 2.6$ that seemed to be independent of changes in the flux level. EGRET observations at $\Theta=0.4$, like MAGIC observations, show a second broad peak at $\Phi \simeq 0.5$, whereas EGRET observations at $\Theta=0.18$ show only a peak at periastron passage. INTEGRAL observations give $\Gamma=3.6_{-1.1}^{+1.6}$ where we find $\alpha<0$ and a steep power-law is expected. In particular, the radio spectral index, $\alpha \simeq-0.5$, we determined for $\mathrm{LS} \mathrm{I}+61^{\circ} 303$, corresponds to an electron power law index $p \simeq 2$ and implies high energy emission, by inverse Compton process, with a photon index $\Gamma>2.2$.

6. Where we have $\alpha \geq 0$, INTEGRAL observations give $\Gamma=1.4-1.7$, as is expected for the low/hard X-ray state. Outside the major outburst window, the X-ray and radio luminosities of LS I $+61^{\circ} 303$ fulfill the universal relationship existing between $L_{\mathrm{X}}$ and $L_{\mathrm{R}}$ in the low/hard X-ray state. The low X-ray luminosity and the well established presence of optical emission, related to the orbital period and to the orbital occurrence of the radio emission, strongly indicate that LS I $+61^{\circ} 303$ is between a low/hard and a quiescent state.

On the basis of these results we conclude that the object LS I +61 303 in a nearly quiescent $\mathrm{X}$-ray state - with $\Gamma \simeq 1.4-1.7$ and with a core centered slow jet with radio spectral index $\alpha \geq 0$ - evolves twice along the $26.5 \mathrm{~d}$ orbit into a steep power-law X-ray state. This last state is related to an unbroken steep power-law with $\Gamma \simeq 2.5$ and to a transient jet with radio spectral index $\alpha<0$. The length of the quiescent state phase strongly depends upon the 1667 d cycle.

Our work indicates that the radio spectral index, $\alpha$, is a powerful tool to distinguish between classes of ejection and also that a periodic radio source, such as LS I $+61^{\circ} 303$, represents an unique possibility for future investigations on the physics of the shock associated to the transient jet. Concerning the relation of gamma-ray emission and transient jet, the first phase of the shock, the growth stage, clearly is of particular interest, where the dominant cooling mechanism is inverse Compton losses. Future observations should confirm whether these Compton losses are responsible for the unbroken steep power law. 
We are grateful to Russ Taylor, Alan Roy, Karl M. Menten, Jürgen Neidhöfer, and Simon Vidrih for their comments. The Green Bank Interferometer is a facility of the National

Science Foundation operated by the NRAO in support of NASA High Energy Astrophysics programs.

\section{REFERENCES}

cciari, V. A., et al. 2009, ApJ, 700, 1034

Albert, J. et al. 2006, Science, 312, 1771

Albert, J., et al. 2008a, ApJ, 684,1351

Albert, J., et al. 2008b, astro-ph/0806.1865

Bosch-Ramon, V., \& Paredes, J. M. 2004, A\&A, 425, 1069

Bosch-Ramon, V., Paredes, J. M., Romero, G. E., \& Ribó, M. 2006, A\&A, 459, L25

Brocksopp, C., Jonker, P. G., Fender, R. P., Groot, P. J., van der Klis, M., \& Tingay, S. J. 2001, MNRAS, 323, 517

Casares, J., Ribas, I., Paredes, J. M., Martí, J., \& Allende Prieto, C. 2005, MNRAS, 360, 1105

Chernyakova, M., Neronov, A., \& Walter, R. 2006, MNRAS, 372, 1585

Combi, J. A., Ribó, M., Mirabel, I. F., \& Sugizaki, M. 2004, A\&A, 422, 1031

Connors, T. W., Johnston, S., Manchester, R. N., \& McConnell, D. 2002, MNRAS, 336, 1201

Dhawan, V., Mirabel, I. F., \& Rodríguez, L. F. 2000, ApJ, 543, 373

Dhawan, V., Muno, M., \& Remillard, R. 2005, ASP Conference Proceedings, 340, 276

Dhawan, V., Mioduszewski, A., \& Rupen, M. 2006, Proceedings of the VI Microquasar Workshop, p. 52.1

Falcke, H., Körding, E., \& Markoff, S. 2004, A\&A, 414, 895

Fender, R. P., Garrington, S. T., McKay, et al. 1999, MNRAS, 304, 865 
Fender, R. P., Pooley, G. G., Durouchoux, P., Tilanus, R. P. J., \& Brocksopp, C. 2000, MNRAS, 312, 853

Fender, R. P. 2001, MNRAS, 322, 31

Fender, R. P., Gallo, E., \& Jonker, P. G. 2003, MNRAS, 343, L99

Fender, R. P., Belloni, T. M., \& Gallo, E. 2004, MNRAS, 355, 1105

Fender, R. P., Stirling, A. M., Spencer, R. E., Brown, I., Pooley, G. G., Muxlow, T. W. B., \& Miller-Jones, J. C. A. 2006, MNRAS, 369, 603

Gallo, E., Fender, R. P., \& Pooley, G. G. 2003, MNRAS, 344, 60

Gallo, E., Fender, R., Kaiser, C., Russell, D., Morganti, R., Oosterloo, T., \& Heinz, S. 2005, Nature, 436, 819

Gallo, E., Fender, R. P., Miller-Jones, J. C. A., et al. 2006, MNRAS, 370, 1351

Gregory, P. C. 2002, ApJ, 575, 427

Gregory, P. C., Neish, C. 2002, ApJ, 580, 1133

Grove, J. E., Johnson, W. N, Kroeger, R. A, et al. 1998, ApJ, 500, 899

Grundstrom, E. D., et al. 2007, ApJ, 656, 437

Han, X., \& Hjellming, R. M. 1992, ApJ, 400, 304

Hannikainen, D. C., Wu, K., Stevens, J. A., Vilhu, O., Rodriguez, J., Hjalmarsdotter, L., \& Hunstead, R. W. 2006, Chinese Journal of Astronomy and Astrophysics Supplement, 6, 010000

Harrison, F. A., Ray, P. S., Leahy, D.A., Waltman, E. B., \& Pooley, G. G. 2000, ApJ, 528, 454

Hutchings, J. B., Crampton, D. 1981, PASP, 93, 486

Jonker, P. G., Nelemans, G., \& Bassa, C. G. 2007, MNRAS, 374, 999

Kaiser, C. R., Sunyaev, R., \& Spruit, H. C. 2000, A\&A, 356, 975

Lindfors, E. J., et al. 2006, A\&A, 456, 895

Malzac, J., Lubiński, P., Zdziarski, A. A., Cadolle Bel, M., Türler, M., \& Laurent, P. 2008, A\&A, 492, 527 
Marscher, A. P., \& Gear, W. K. 1985, ApJ, 298, 114

Marscher, A. P., et al. 2008, Nature, 452, 966

Martí, J., \& Paredes, J. M. 1995, A\&A, 298, 151

Massi, M., Ribó, M., Paredes, J. M., Garrington, S. T., Peracaula, M., \& Martí, J. 2005, High Energy Gamma-Ray Astronomy, 745, 311

Massi, M. 2007, The Multicolored Landscape of Compact Objects and Their Explosive Origins, 924,729

McClintock, J. E., \& Remillard, R. A. 2003, astro-ph/0306213

McClintock, J. E., \& Remillard, R. A. 2006, Compact stellar X-ray sources, Cambridge University Press, p. 157,

McClintock, J. E., Narayan, R., Garcia, M. R., et al. 2003, ApJ, 593, 435

McConnell, M. L., et al. 2001, Gamma 2001: Gamma-Ray Astrophysics, 587, 96

Mendelson, H., \& Mazeh, T. 1989, MNRAS, 239, 733

Mendelson, H., \& Mazeh, T. 1994, MNRAS, 267, 1

Merloni, A., Heinz, S., \& di Matteo, T. 2003, MNRAS, 345, 1057

Merloni, A., \& Heinz, S. 2007, MNRAS, 381, 589

Murdin, P., Jauncey, D. L., Lerche, I., et al. 1980, A\&A, 87, 292

Narayan, R., Barret, D., \& McClintock, J. E. 1997, ApJ, 482, 448

Paredes, J. M., Marti, J., Estalella, R., \& Sarrate, J. 1991, A\&A, 248, 124

Paredes, J. M., et al. 1994, A\&A, 288, 519

Paredes, J. M., Martí, J., Peracaula, M., Ribo, M. 1997, A\&A, 320, L25

Paredes, J. M., Ribó, M., Ros, E., Martí, J., \& Massi, M. 2002, A\&A, 393, L99

Peracaula, M., Martí, J., \& Paredes, J. M. 1997, A\&A, 328, 283

Pooley, G. G., \& Fender, R. P. 1997, MNRAS, 292, 925

Remillard, R. A., \& McClintock, J. E. 2006, ARA\&A, 44, 49 
Rodriguez, L. F., \& Mirabel, I. F. 1997, ApJ, 474, L123

Romero, G. E., Okazaki, A. T., Orellana, M., \& Owocki, S. P. 2007, A\&A, 474, 15

Russell, D. M., Fender, R. P., Hynes, R. I., Brocksopp, C., Homan, J., Jonker, P. G., \& Buxton, M. M. 2006, MNRAS, 371, 1334

Russell, D. M., Fender, R. P., \& Jonker, P. G. 2007, MNRAS, 379, 1108

Tavani, M., Kniffen, D., Mattox, J. R., Paredes, J. M., \& Foster, R. 1998, ApJ, 497, L89

Taylor, A. R., \& Gregory, P. C. 1982, ApJ, 255, 210

Taylor, A. R., Kenny, H. T., Spencer, R. E., \& Tzioumis, A. 1992, ApJ, 395, 268

Taylor, A. R., Dougherty, S. M., Scott, W. K., Peracaula, M., \& Paredes, J. M. 2000, proc. of Astrophysical Phenomena Revealed by Space VLBI, eds. H. Hirabayashi, P. G. Edwards, and D. W. Murphy, pub. ISAS, p. 223

Taylor, A. R., Dougherty, S. M., Scott, W. K., Peracaula, M., \& Paredes, J. M. 2001, Galaxies and their Constituents at the Highest Angular Resolutions, 205, 270

Türler, M., Courvoisier, T. J.-L., \& Paltani, S. 2000, A\&A, 361, 850

Türler, M., Courvoisier, T. J.-L., Chaty, S., \& Fuchs, Y. 2004, A\&A, 415, L35

Tudose, V., Soleri, P., Fender, R. P., et al. 2006, Proceedings of the VI Microquasar Workshop, p.98.1

Valtaoja, E., Terasranta, H., Urpo, S., Nesterov, N. S., Lainela, M., \& Valtonen, M. 1992, A\&A, 254, 71

Van der Laan, H. 1966, Nature, 211, 1131

Zaitseva, G. V., \& Borisov, G. V. 2003, Astronomy Letters, 29, 188

Zamanov, R. K, \& Martí, J. 2000, A\&A, 358, L55 


\section{LS I +61303}

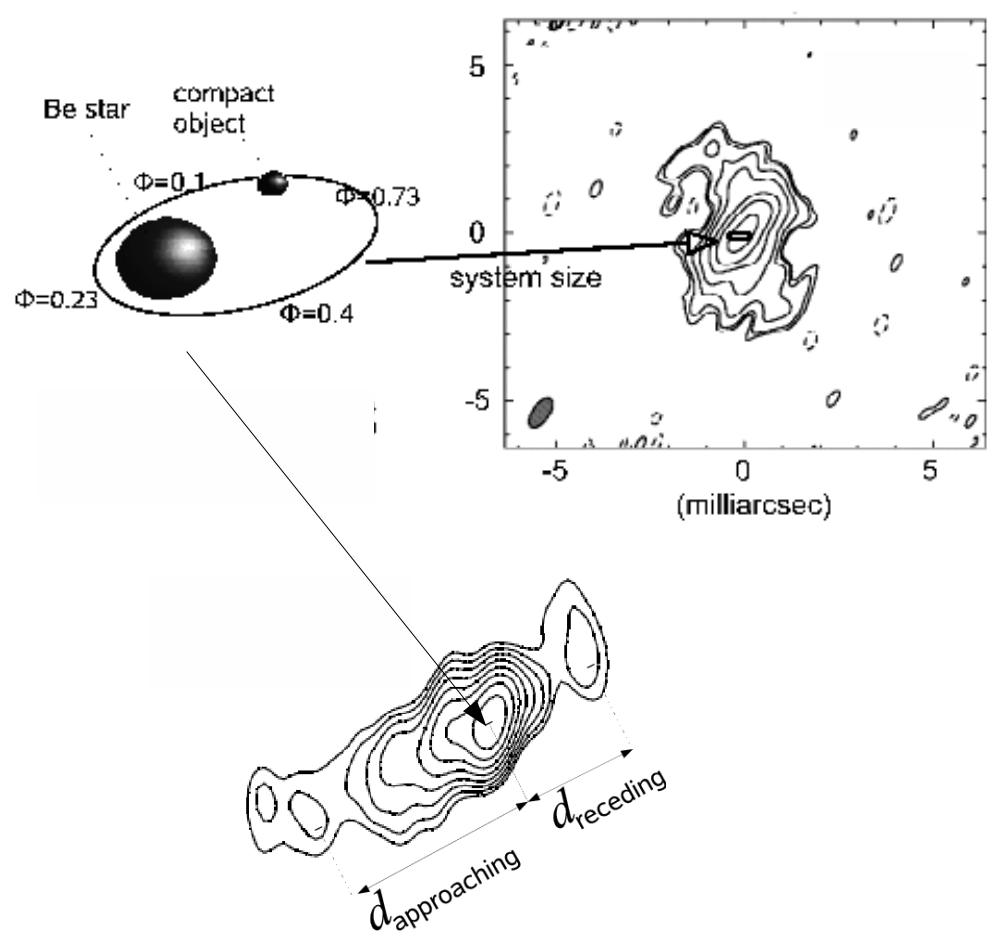

Fig. 1. - Top-left: Sketch of the binary system. Top-right: Radio observation by Taylor et al. (2000). The resolution of the radio image is $\sim 0.95$ mas $\times 0.45$ mas and is shown in the left bottom corner as a grey ellipse. The dimension of the orbit is drawn in the center of the radio source. Bottom: VLBA image by Dhawan et al. (2006). The resolution of the radio image is $\sim 1.5$ mas $\times 1.1$ mas, therefore, as the previous image, insufficient to distinguish any variations at the much smaller orbital scale. The image can be interpreted as a steady jet (Sec. 4.2) with asymmetries due to relativistic Doppler effects, i.e. with the receding jet shorter than the approaching one. Consistently, also the approaching and receding plasmoids at both sides are at different distances $\left(d_{a} \neq d_{r}\right)$. 

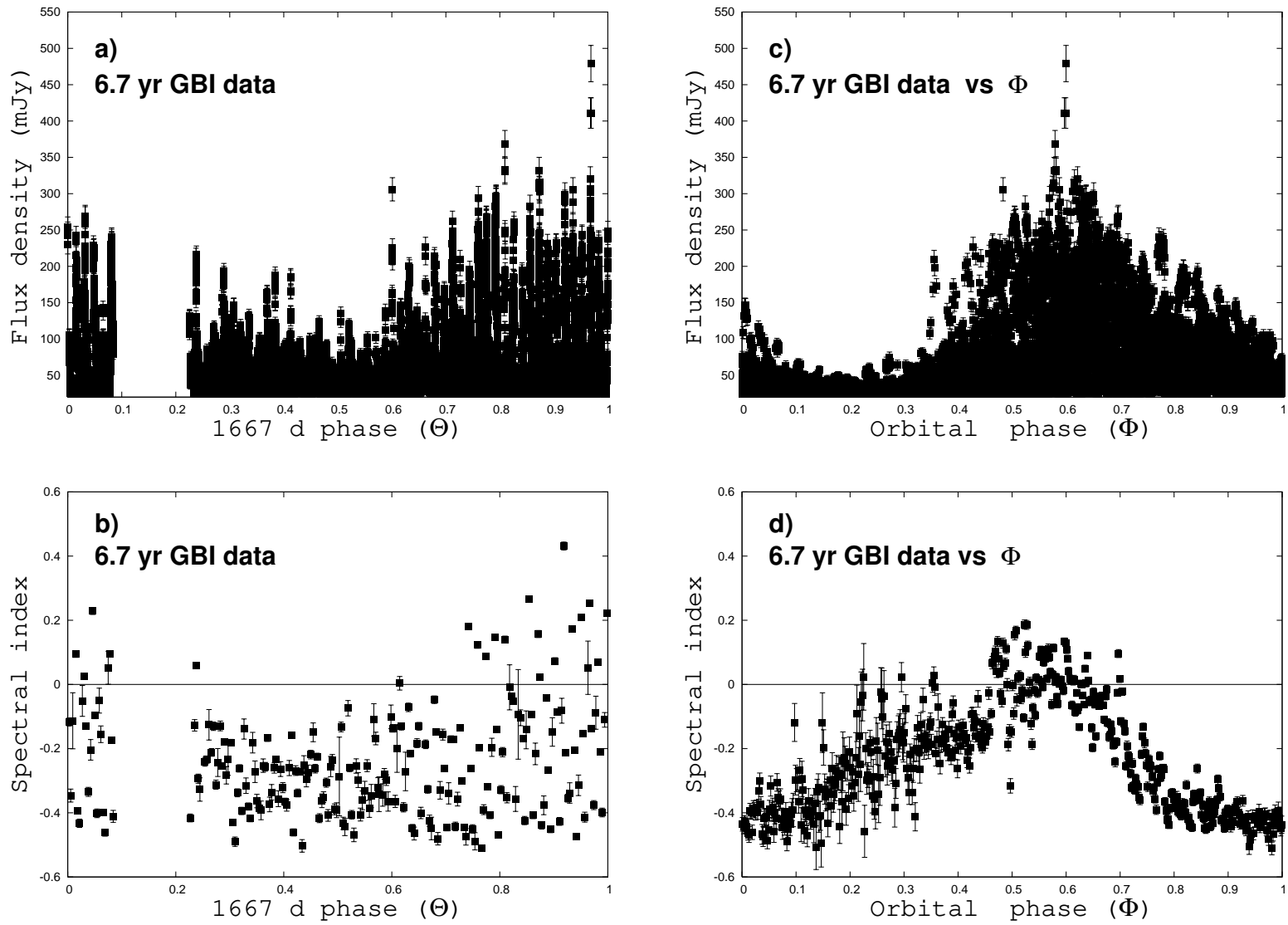

Fig. 2.- a: Radio light curve $(8.3 \mathrm{GHz})$ vs $\Theta$, where $\Theta$ is related to $\frac{\left(t-t_{0}\right)}{P_{2}}$ with $t_{0}=$ JD2443366.775 and period $P_{2}=1667 \mathrm{~d}$ (Gregory 2002). The maximum of the cycle is at $\Theta \sim 0.97$. b: Spectral index vs $\Theta$. We averaged the folded spectral index curve binning the data into phase intervals of $\Delta \Theta=0.004$ (equal to $7 \mathrm{~d}$ ). c: Radio light curve vs $\Phi$, the orbital phase, with $\Phi$ related to $\frac{\left(t-t_{0}\right)}{P_{1}}$, with $P_{1}=26.496 \mathrm{~d}$ (Gregory 2002) d: Spectral index vs $\Phi$, averaged over $\Delta \Phi=0.002,(\sim 1.3 \mathrm{~h})$ 

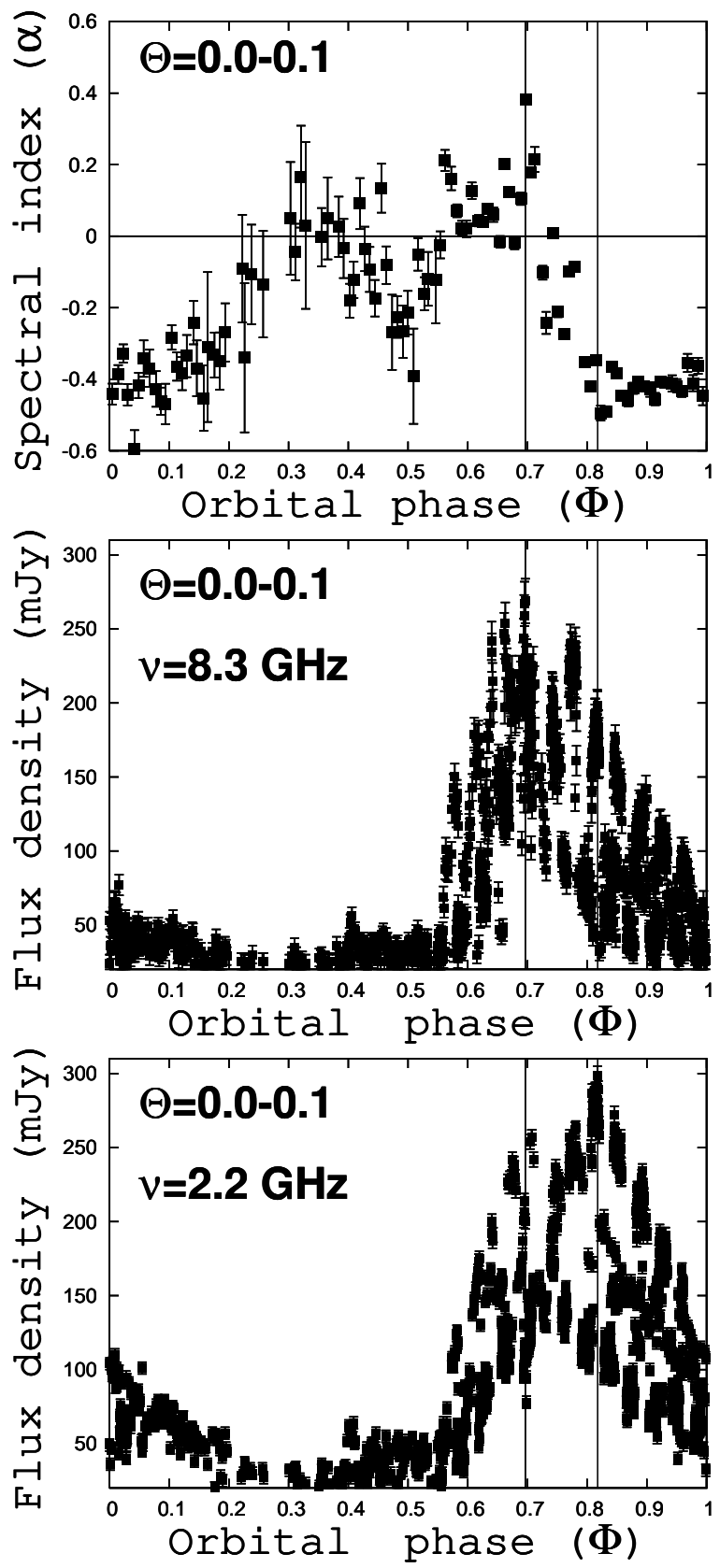

Fig. 3.- Spectral index and flux density, at $8.3 \mathrm{GHz}$ and $2.2 \mathrm{GHz}$ vs orbital phase, $\Phi$, for the GBI data in the interval $\Theta=0.0-0.1$. The spectral index, $\alpha$, is averaged over $\Delta \Phi=0.009$, $(\sim 6 \mathrm{~h})$. Note that the evolution from an optically thick to an optically thin spectrum occurs twice, giving the $\alpha$ vs $\Theta$ curve a double-peaked shape. The radio outburst peaks first at 8.3 GHz, then it follows a larger peak at $2.2 \mathrm{GHz}$. Between the two outbursts there is an inversion of the spectrum from inverted to optically thin. 

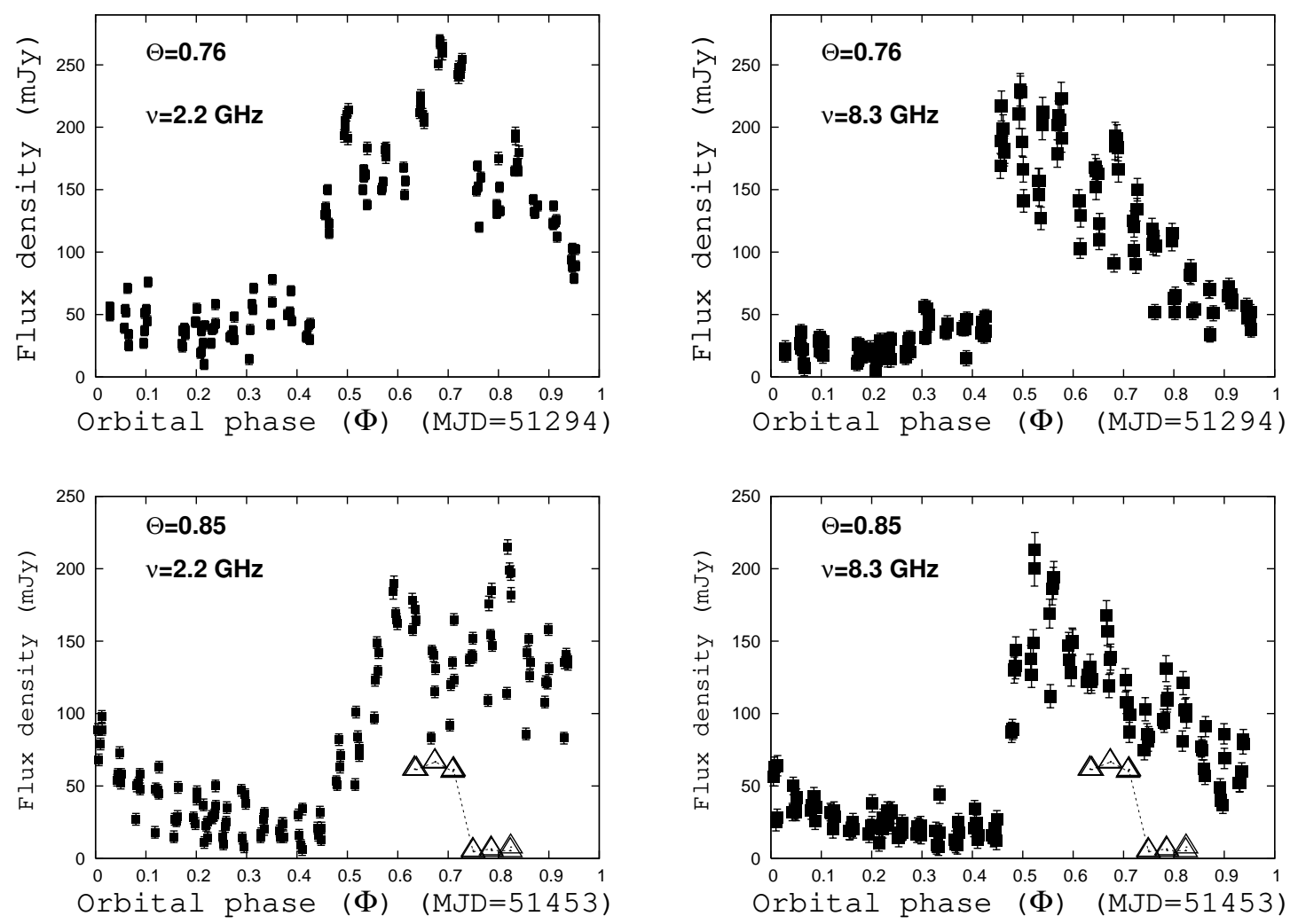

Fig. 4. - Top: Light curve at $2.2 \mathrm{GHz}$ and $8.3 \mathrm{GHz}$ at $\Theta=0.76$. Both light curves at $2.2 \mathrm{GHz}$ and $8.3 \mathrm{GHz}$ have two peaks: there are two consecutive outbursts, one with optically thick spectrum and the second with optically thin spectrum. The relative spectral index is shown in Fig. 5, panel 7. Bottom: Light curves at $\Theta=0.85$. The relative spectral index is shown in Fig. 5, panel 8. The triangles are the $\mathrm{H} \alpha$ emission-line measurements by Grundstrom et al. (2007, Fig. 3-Bottom) multiplied by a factor of -5 to fit in the plot. The $\mathrm{H} \alpha$ value, still high at $\Phi=0.71$, registers a dramatic decline at $\Phi=0.749$ (1 day later) in correspondence with the onset of the optically thin outburst. 

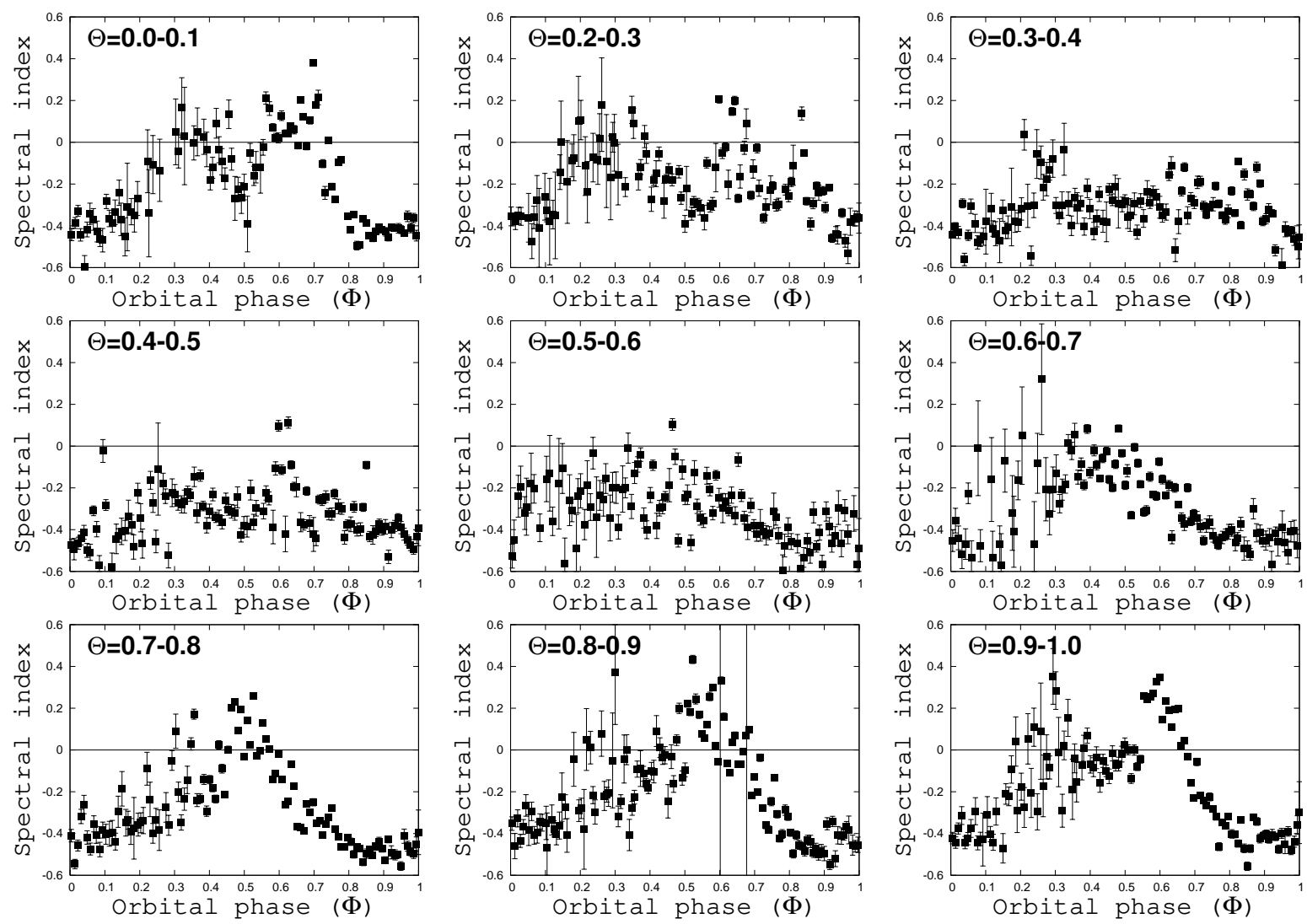

Fig. 5.- Spectral index vs orbital phase, i.e. $\alpha$ vs $\Phi$, for different intervals of $\Theta$. The spectral index is averaged over $\Delta \Phi=0.009(\sim 6 \mathrm{~h})$. During $\Theta=0.0-0.3$ and $\Theta=0.7-1.0$, i.e. during the maximum of the $1667 \mathrm{~d}$ cycle, two optically thick intervals are present, i.e. intervals along the orbit where $\alpha \geq 0$. The two optically thick intervals are from $\Phi=0.22 \pm 0.08$ to $\Phi=0.33 \pm 0.12$ for the first one and, from $\Phi=0.50 \pm 0.09$ to $\Phi=0.70 \pm 0.13$ for the second one. The interval $\Theta=0.8-0.9$ contains two vertical lines showing the period when the GBI data were simultaneous with the VSOP observations by Taylor et al. (2001). 

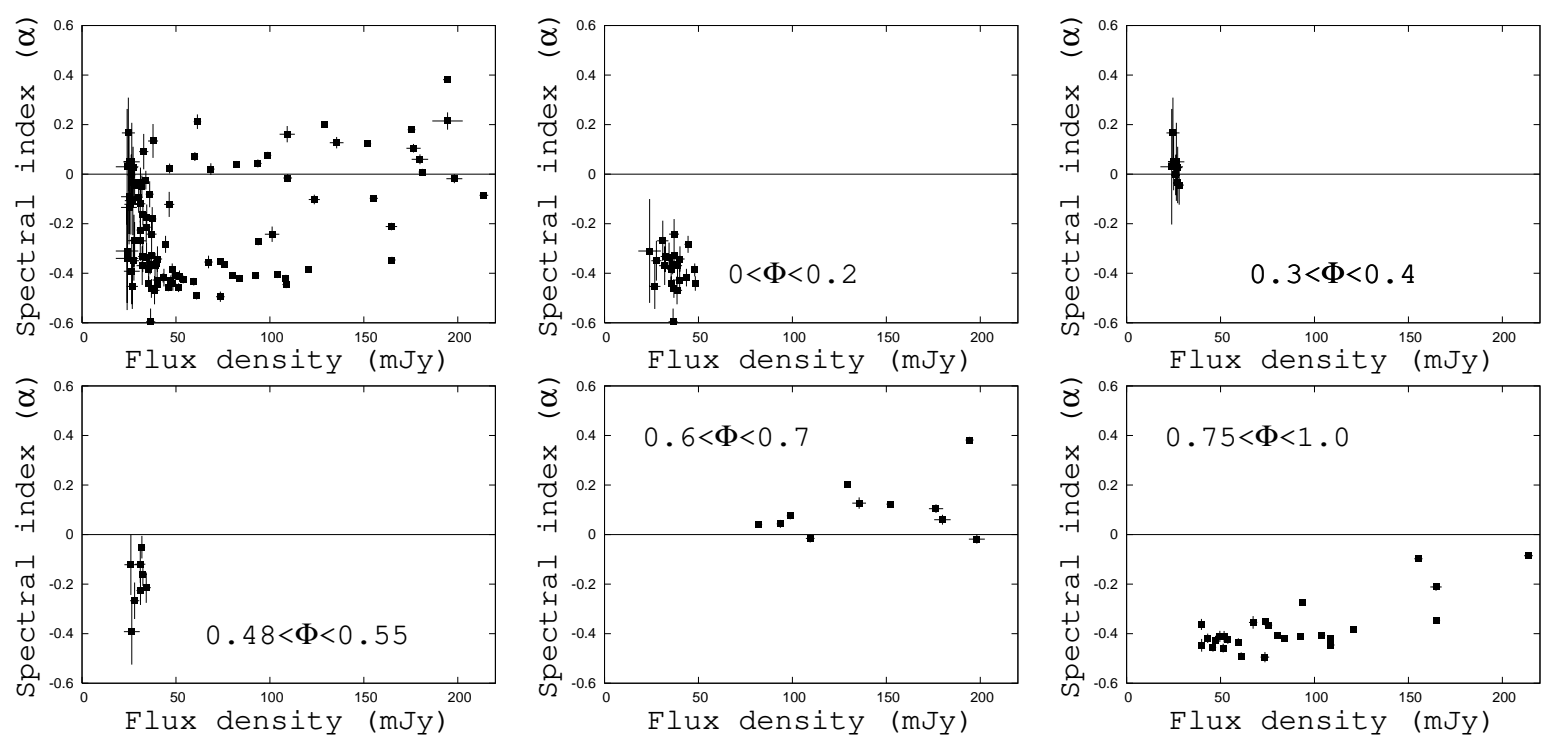

Fig. 6. - Spectral index vs averaged density flux $(8 \mathrm{GHz})$ for the data set of Fig. 3. The first plot covers $\Phi$ from 0.0 to 1.0, whereas the other plots select different orbital phase intervals. The first optically thick interval centered at $\Phi \sim 0.35$ is associated to low flux density whereas the second optically thick interval, centered at $\Phi \sim 0.65$, is associated with high flux density. 

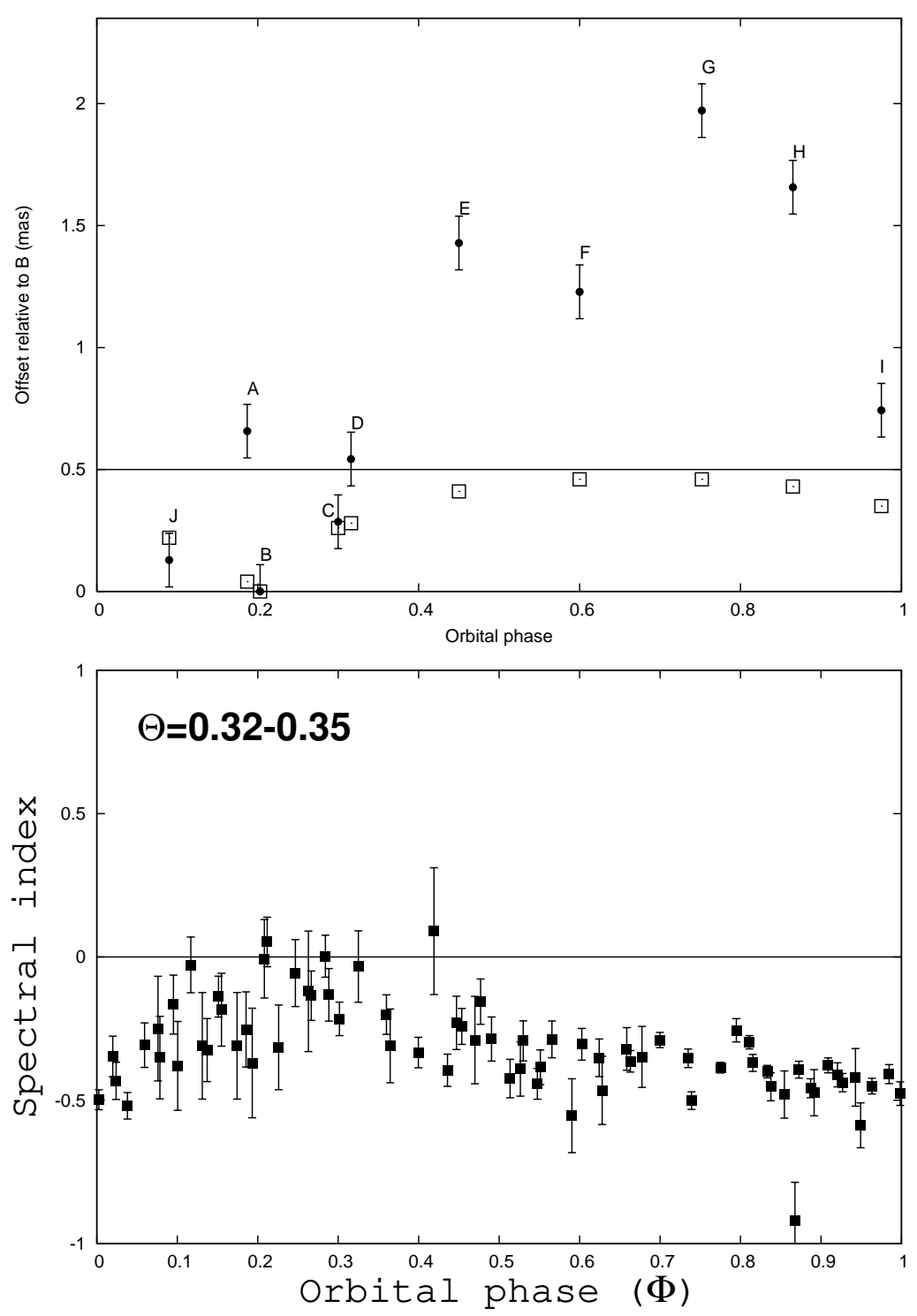

Fig. 7.- Top: Quantitative comparison of the astrometry of the radio peaks presented by Dhawan et al. (2006) with respect to the position of the compact object along the orbit. Offsets relative to the radio peak B $(\Phi=0.203)$ from Dhawan et al. (2006) vs. orbital phase are shown by black dots. The offset is shown with the error bars of 0.1 mas as given by these authors. The empty squares show the distance in the orbit from phase $\Phi=0.203$ to all the other phases, where the VLBA observations have been performed. These relative distances have been computed using the orbit from Casares et al. (2005). The horizontal line at 0.5 mas indicates the major axis, i.e. the maximum possible distance in the orbit. Bottom: spectral index vs orbital phase (average over $\sim 7 \mathrm{~h}$ ) for a subset of the GBI data base at different epochs than Dhawan et al. (2006) observations, but covering the same phase $(\Theta=0.32-0.35)$ with respect to the long $1667 \mathrm{~d}$ cycle. 